\title{
Gram-Scale Synthesis of Tetrahedrite Nanoparticles and Their
}

\section{Thermoelectric Properties}

Takeshi Nakada,${ }^{1}$ Mari Takahashi,${ }^{1}$ Chiko Shijimaya,${ }^{1}$ Koichi Higashimine,${ }^{2}$ Wei Zhou,${ }^{1}$ Pratibha Dwivedi,${ }^{1}$ Michihiro Ohta ${ }^{3}$ Hiroshi Takida,${ }^{4}$ Takeo Akatsuka,${ }^{4}$ Masanobu Miyata,${ }^{1}$ and Shinya Maenosono ${ }^{1 *}$

${ }^{1}$ School of Materials Science, Japan Advanced Institute of Science and Technology, 1-1 Asahidai, Nomi, Ishikawa 923-1292, Japan

${ }^{2}$ Center for Nano Materials and Technology, Japan Advanced Institute of Science and Technology, 1-1 Asahidai, Nomi, Ishikawa 923-1292, Japan

${ }^{3}$ Research Institute for Energy Conservation, National Institute of Advanced Industrial Science and Technology (AIST), Tsukuba, Ibaraki 308-8568, Japan

${ }^{4}$ Research Center, Nippon Shokubai Co., Ltd., Himeji, Hyogo 671-1292, Japan

*E-mail: shinya@jaist.ac.jp 

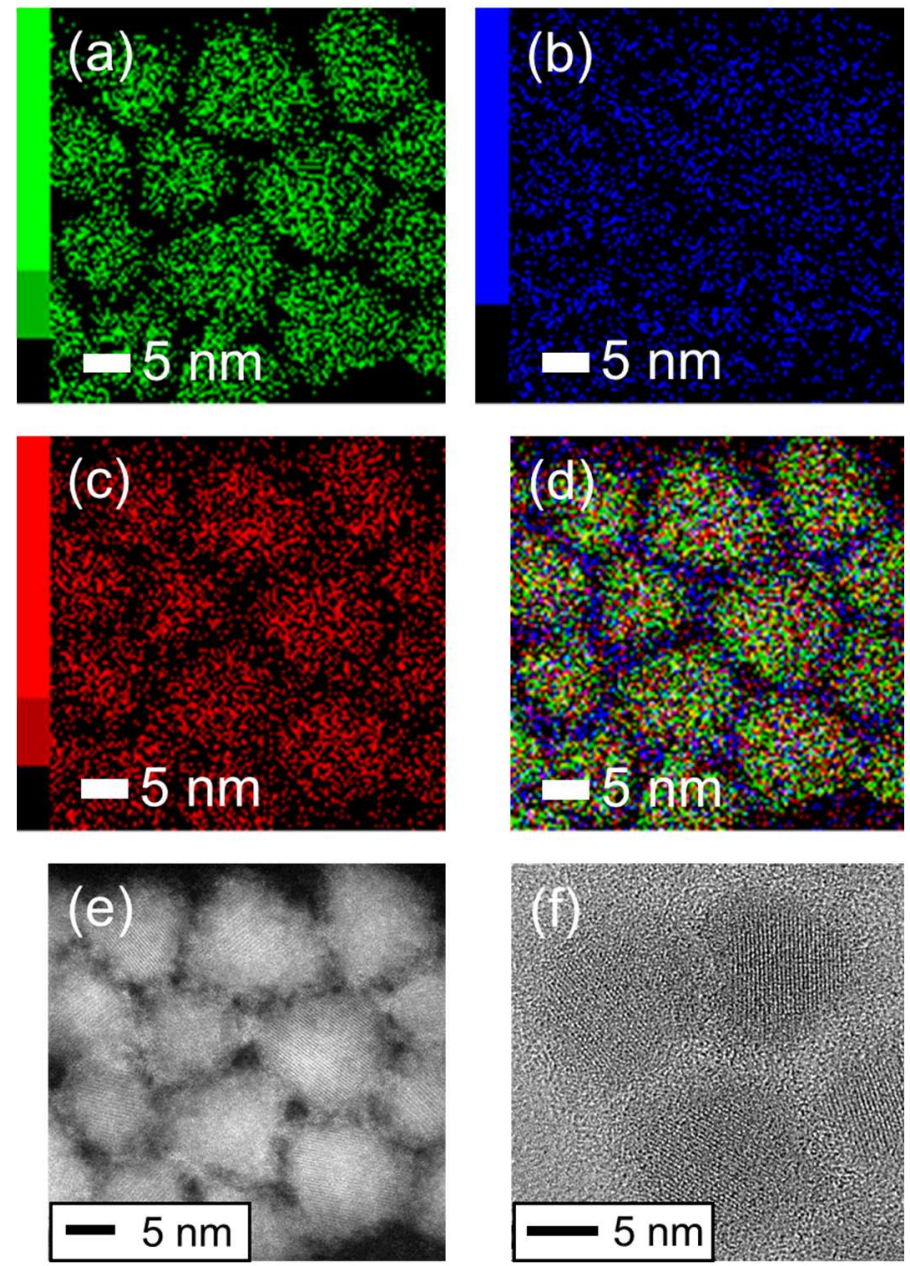

Figure S1. (a) Cu K-line, (b) Sb L-line, (c) S K-line, and (d) merged images of the EDS elemental mapping images of the S-NPs. (e) HAADF-STEM and (f) HR-TEM images of the S-NPs.
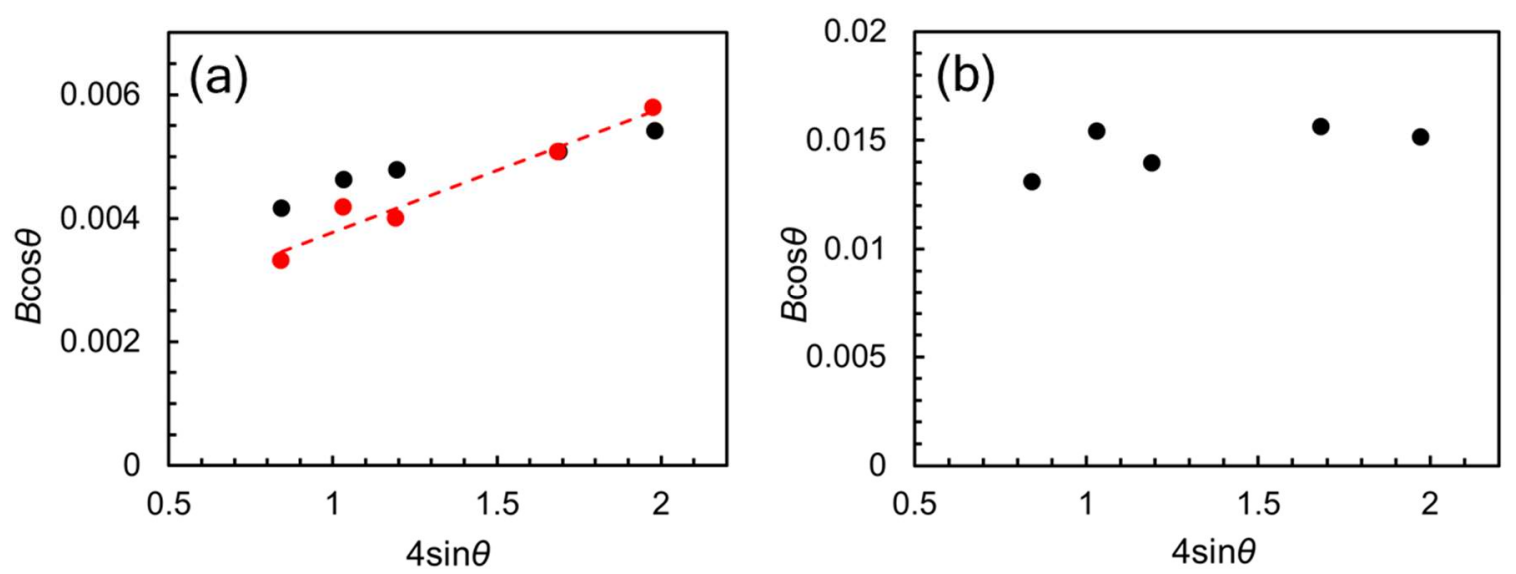

Figure S2. W-H plots of the (a) L-NPs (black) and L-pellet (red) and (b) S-NPs. 

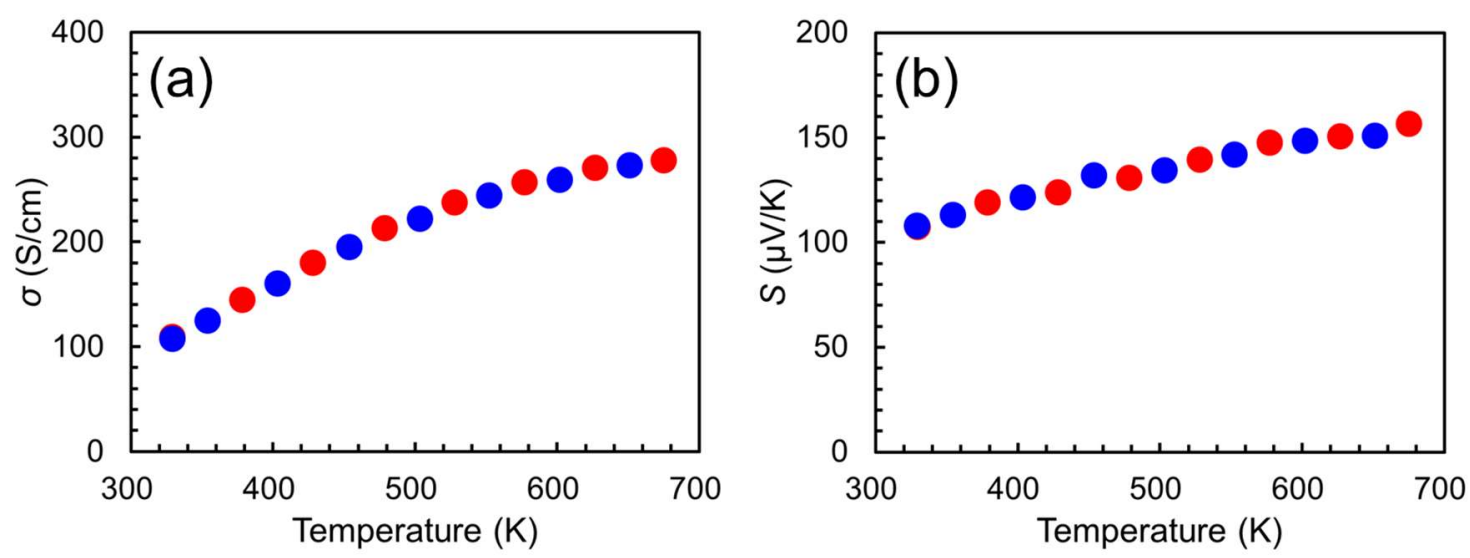

Figure S3. (a) $\sigma$ and (b) $S$ as a function of temperature. The red and blue circles represent the data measured in the heating and cooling cycles, respectively.

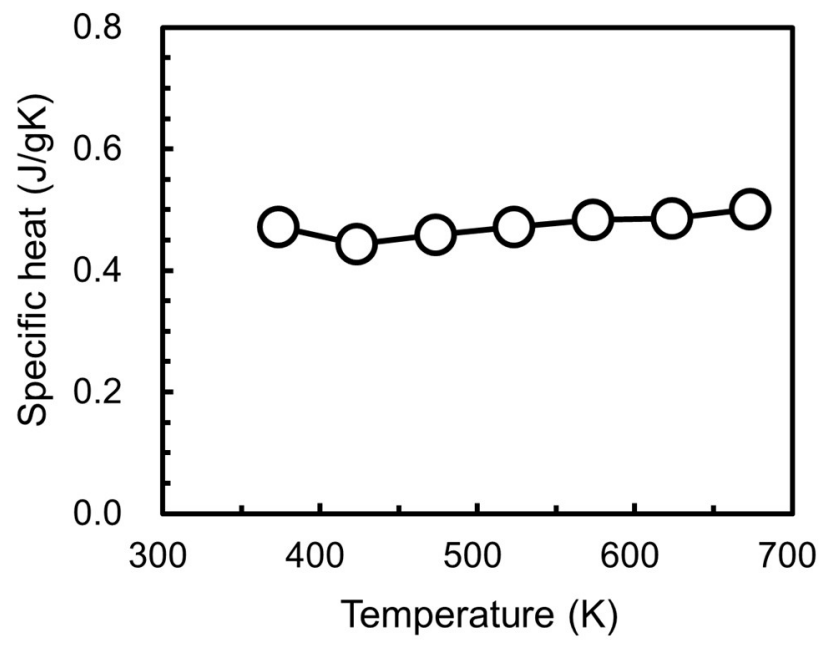

Figure S4. Specific heat $\left(\mathrm{J} \cdot \mathrm{g}^{-1} \cdot \mathrm{K}^{-1}\right)$ of the L-pellet. 